\title{
Signal Detection
}

\section{OVERVIEW}

The bulk magnetization $M_{0}$ represents the equilibrium value of the magnetization when all spins have aligned themselves parallel to the main field. When this magnetization is tipped into the transverse plane, it precesses about the main field. This precession generates a changing flux through the face of an appropriately placed coil inducing an electromotive force (emf) in the coil. The measured signal is proportional to the induced emf.

The emf is the negative time derivative of the magnetic flux and can be calculated by Faraday's law of induction. The magnetic flux represents how much magnetic field cuts through the area of the pick-up coil. Consider a rotating field through a square loop (Fig. B2.1.1B). This case is close to the usual MR case where the transverse magnetization precesses about the main field $B_{0}$ at the Larmor frequency. The actual flux and its emf are calculated in the technical discussion. Because the spin precesses very rapidly (recall the Larmor frequency is $42.6 \mathrm{MHz}$ at $1 \mathrm{~T}$ ), the longitudinal magnetization contributes negligibly to the change in flux per unit time. It is the precession of the magnetization $M_{0}$ through the coil that induces the main signal, not the $T_{1}$ or $T_{2}$ relaxation of $M_{0}$; however, the coupling of $M_{0}$ to the coil does induce relaxation as well, but this is beyond the scope of this text.

Since the signal oscillates at the Larmor frequency, a demodulation of the signal is needed in order to more easily examine the MR signal. Demodulation corresponds to the multiplication of the signal by a sinusoid or cosinusoid with a frequency at or near the Larmor frequency $\omega_{0}$. If the sample is uniform and the MR system is perfect, the signal will oscillate exactly at the Larmor frequency. A demodulation signal is applied at the frequency $\omega_{0}+\delta \omega$. After the demodulation, two frequencies appear, one at $\delta \omega$ and the other one at $2 \omega_{0}+\delta \omega$ (see Fig. B2.1.2). Then a low-pass filter is applied to remove the signal at the higher frequency. In MR measurements, signals are demodulated and filtered before any image reconstruction is performed. (Usually, when people refer to the demodulated signal, they mean the demodulated and filtered signal.) After Fourier-transforming the signal, an MR imaging is created. Demodulating the signal is equivalent to looking at the signal in the rotating reference frame.

A simple example of detecting an MR signal is the free induction decay (FID) experiment. A $\pi / 2$-pulse tips the spin into the transverse plane where it dephases or decays (the so-called $T_{2}$ decay, when no field inhomogeneities are present). An FID is performed routinely on MRI machines to adjust the central frequency and optimize system response. Figure B2.1.3 gives four examples of FID experiments and demodulations. In Figure B2.1.3A, the RF field and the signal are shown in the laboratory reference frame. The signal has a rapid oscillation with a $T_{2}$ exponential decay envelope. Figure $\mathrm{B} 2.1 .3 \mathrm{~B}$ shows the RF field and the signal in the rotating frame-i.e., a demodulated signal. If the signal is not demodulated at the Larmor frequency, an oscillation still exists. This is shown in Figure B2.1.3C. Finally, if the total signal is from an ensemble of spins, and each spin precesses at frequencies slightly different from the Larmor frequency, then the total signal will decay faster than the original $T_{2}$ decay. This is called $T_{2}{ }^{*}$ decay and is shown in Figure B2.1.3D.

If we consider an ideal MR system, the signal $s(t)$ will be proportional to the product of the Larmor frequency, magnetization, and object size $\left(V_{s}\right)$ confined by the field-of-view, i.e., $s(t) \propto \omega_{0} M_{0} V_{s}$. This implies that the signal is proportional to the square of the static

Radiofrequency Excitation and Reception

B2.1.1 
Table B2.1.1 Relative Signal Strength of Different Nuclei ${ }^{a}$

\begin{tabular}{llllll}
\hline Nucleus $i$ & $\nvdash_{i}(\mathrm{MHz} / \mathrm{T})$ & $r_{i}$ & $a_{i}$ & $s_{i}$ & $\mathcal{R}_{i} / \mathcal{R}\left({ }^{1} \mathrm{H}\right)$ at $1 \mathrm{~T}$ \\
\hline${ }^{1} \mathrm{H}$, gray matter & 42.5764 & 1.0 & 1.0 & $1 / 2$ & 1.0 \\
${ }^{23} \mathrm{Na}$, average tissue & 11.2686 & $9.1 \times 10^{-4}$ & 1.0 & $3 / 2$ & $8.4 \times 10^{-5}$ \\
${ }^{31} \mathrm{P}$, average tissue & 17.2510 & $8.5 \times 10^{-4}$ & 1.0 & $1 / 2$ & $5.7 \times 10^{-5}$ \\
${ }^{17} \mathrm{O}$, gray matter & -5.7741 & 0.5 & $3.7 \times 10^{-4}$ & $5 / 2$ & $5.4 \times 10^{-6}$ \\
${ }^{19} \mathrm{~F}$, average tissue & 40.0765 & $4.5 \times 10^{-8}$ & 1.0 & $1 / 2$ & $3.8 \times 10^{-8}$
\end{tabular}

${ }^{a}$ Table of $\gamma$, fraction of all isotopes $r$ relative to ${ }^{1} \mathrm{H}$ in gray matter, natural abundance $a$, spin $s$ in units of $\hbar$ and calculated relative sensitivity of other elements of interest. The values presented refer to a fixed field strength. Note that a negative value of $₹$ means that precession occurs in the counterclockwise direction. The product of $a_{i} r_{i}$ and the molarity $88 \mathrm{M}$ for

${ }^{1} \mathrm{H}$ in gray matter yields the relative body abundances of Table B1.1.1.

field strength, since both $\omega_{0}$ and $M_{0}$ depend linearly on $B_{0}$; however, at high fields, noise is proportional to the static field strength, so the signal-to-noise ratio is only linearly proportional to the static field strength. The reason we image protons in the human body using MRI is because almost all tissues have a very high water content. Other elements that are MR active, such as sodium or phosphorus, are much less abundant. The relative sensitivities of those elements to the water content of gray matter are listed in the last column of Table B2.1.1.

\section{TECHNICAL DISCUSSION}

In Chapter B1, we discussed the spin behavior in a static magnetic field. We introduced the bulk magnetization $M_{0}$ and an RF field to tip this magnetization into the transverse plane. We now turn our focus to how the MR signal is generated. We start with the induced electromotive force (emf) generated by the precessing magnetization by introducing Faraday's law.

\section{Faraday Induction}

The emf induced in a coil by a temporal change in its magnetic flux can be calculated by Faraday's law of induction:

$$
\mathrm{emf}=-\frac{d \Phi}{d t}
$$

where $\Phi$ is the flux through the coil:

$$
\Phi=\int_{\text {coil area }} \vec{B} \cdot d \vec{S}
$$

The vector $d \vec{S}$ has magnitude $d S$ and is normal to the differential area in the direction chosen for the definition of positive flux. The flux can be thought of as being proportional to the number of field lines penetrating the effective area presented by the loops making up the coil. The number of flux lines is a convenient picture, and is arbitrarily normalized. Equation B2.1.2 is, by contrast, well-defined. The currents induced in a conducting loop by a changing flux produce a field which opposes the changes induced by the external field. The result that induced currents produce fields that oppose externally induced flux changes is referred to as Lenz's law.

In the application of Equation B2.1.1 to MRI, a study of elementary examples with wire loops is helpful. The first example is a fixed coil in a time-dependent, fixed-axis magnetic

B2.1.2 
field. Consider a spatially invariant magnetic field making an angle $\theta$ to the normal of the plane of the square coil shown in Figure B2.1.1A. The field is also taken to have a harmonic time dependence with angular frequency $\omega$ and is given by:

$$
\vec{B}(t)=\vec{B} \sin \omega t=B(\sin \theta \hat{y}+\cos \theta \hat{z}) \sin \omega t
$$

for constant $B>0$. The field $\vec{B}(t)$ in Equation B2.1.3 is taken to be uniform over the space of the coil, at any instant in time. (A magnetic field that varies in time must also vary in space, according to Maxwell's equations. However, such spatial variations may be ignored for distances that are small compared to the wavelength, $\lambda=2 \pi c / \omega=c /\left(\psi B_{0}\right)$. At $1.0 \mathrm{~T}$ the free-space wavelength for electromagnetic radiation is $\sim 7 \mathrm{~m}$.) The coil is chosen to lie in the $x-y$ plane so that $\mathrm{d} \vec{S}=d x d y \hat{z}$. From Equations B2.1.1 and B2.1.2, the emf generated in the coil by the time-varying magnetic field is:

$$
\begin{aligned}
\mathrm{emf} & =-\frac{d}{d t} \int_{-L / 2}^{L / 2} d x \int_{-L / 2}^{L / 2} d y \hat{z} \cdot \vec{B}(t) \\
& =-L^{2} B \omega \cos \theta \cos \omega t
\end{aligned}
$$

At $t=0$, for example, the emf is negative, producing a clockwise (negative $\hat{\phi}$ direction) current in the loop. This can be verified by using the right-hand rule to see in which direction the current would have to flow to produce a field that opposes the change in the applied field $\vec{B}(t)$. The appearance of the $\omega$ factor in Equation B2.1.4, which results from the time derivative, is the source of a major enhancement in the MR signal.

In a standard MRI experiment, the field associated with a precessing magnetization sweeps past fixed receiving coils. An example of this scenario is shown in Figure B2.1.1B. The magnetic field rotating about the $x$-axis is given by $\vec{B}(t)=B \cos \omega \mathrm{t} \hat{z}+B \sin \omega \mathrm{t} \hat{y}$ and now $d \vec{S}=d x d y \hat{z}$. The induced emf in this case is

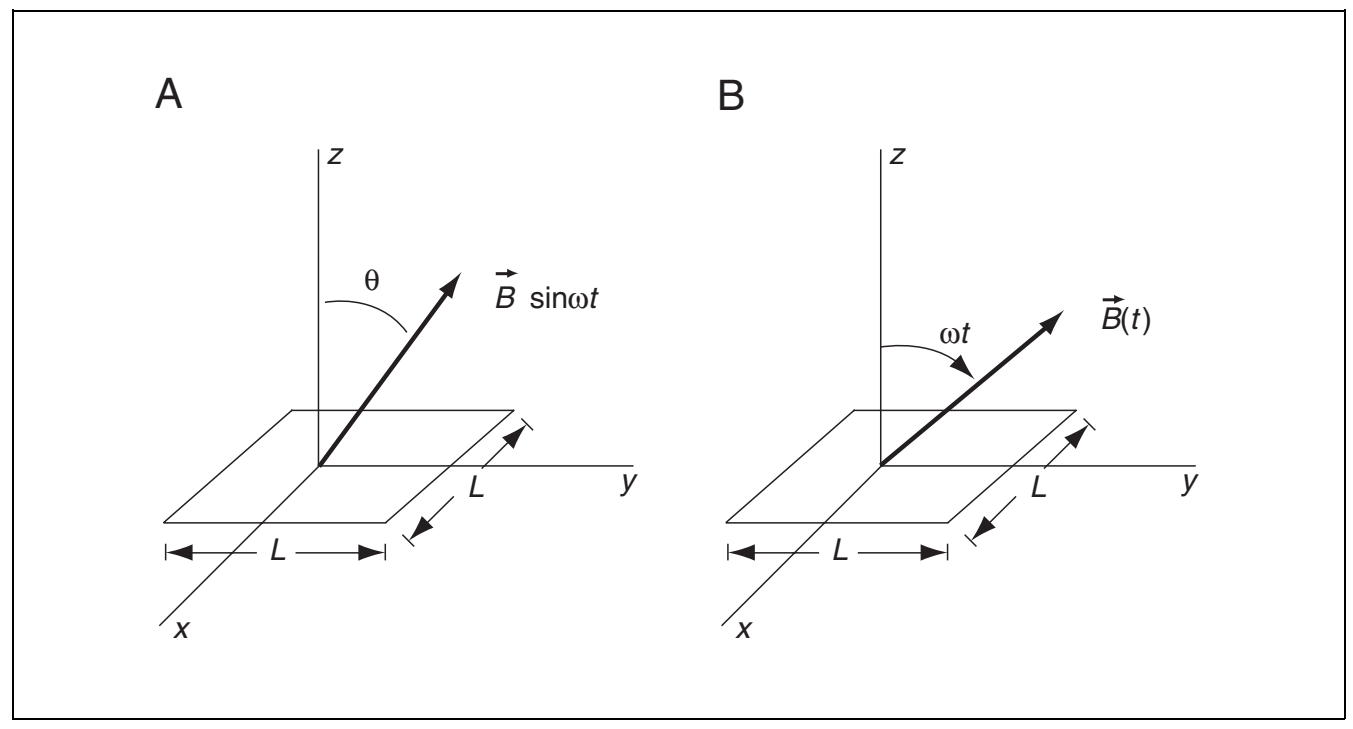

Figure B2.1.1 Two examples of loops of wires experiencing changing flux as a function of time. (A) A square coil is stationary, and the applied field oscillates along a fixed axis. (B) A stationary coil is immersed in a rotating, fixed-magnitude field (an example of special relevance to MR measurements).

Radiofrequency Excitation and Reception

B2.1.3 


$$
\begin{aligned}
\mathrm{emf} & =-\frac{d}{d t} \int_{-L / 2}^{L / 2} d x \int_{-L / 2}^{L / 2} d y \hat{z} \cdot \vec{B}(t) \\
& =L^{2} \omega B \sin \omega t
\end{aligned}
$$

\section{The MRI Signal and the Principle of Reciprocity}

When a magnetic field is applied through a sample, the motion of electrons forms the current flow which causes the magnetization $\vec{M}(\vec{r}, t)$ of the sample. The magnetization of the sample generates a magnetic field which is the induced field due to the applied magnetic field. This induced field is the source of the flux whose emf is picked up by the receive coil of an MR machine. We can define the current normalized receive field as $\overrightarrow{\mathcal{B}}^{\text {receive }}(\vec{r}) \equiv \vec{B}^{\text {receive }}(\vec{r}) / I$, which is the magnetic field per unit current that would be produced by the coil at a given point in space, and derive the flux (see Haacke et al., 1999)

$$
\Phi_{M}(t)=\int_{\text {sample }} d^{3} r \overrightarrow{\mathcal{B}}^{\text {receive }}(\vec{r}) \cdot \vec{M}(\vec{r}, t)
$$

The fact that the flux in Equation B2.1.6 depends upon $\overrightarrow{\mathcal{B}}$ receive $(\vec{r})$, the "receive" field that would be produced by the detection coil at all points where the magnetization is nonzero, is an example of the principle of reciprocity. The original expression as a surface integration over the detection coil area has been replaced by a volume integration over the region of nonzero magnetization. That is, the flux through the detection coil due to the magnetization can be found instead by calculating the flux that would emanate from the detection coil, per unit current, associated with the (rotating) magnetization.

The emf induced in the coil is expressed as:

$$
\begin{aligned}
\text { emf } & =-\frac{d}{d t} \Phi_{M}(t) \\
& =-\frac{d}{d t} \int_{\text {sample }} d^{3} r \vec{M}(\vec{r}, t) \cdot \overrightarrow{\mathcal{B}}^{\text {receive }}(\vec{r})
\end{aligned}
$$

Equation B2.1.7 is a key formula for understanding the factors which affect signal amplitude. The dependence of the emf on the excitation or transmit field $B_{1}{ }^{\text {transmit }}$ is implicit in the dependence of Equation B2.1.7 on the magnetization $\vec{M}(\vec{r}, t)$.

\section{Signal from Precessing Magnetization}

The fundamental signal in an MR experiment comes from the detection of the emf predicted by Equation B2.1.7 for precessing magnetization. In this section, the prediction is analyzed further, in terms of variables involving the static and RF fields, and the properties of the sample of spins. The experiment described below from a given sample is generally referred to as a "free induction decay" (FID). This experiment is discussed in more detail in the next section.

\section{General expression}

It is assumed that the sample is immersed in a static, uniform field $B_{0} \hat{z}$ and has been "excited" by some RF pulse so that there exists, at time $t$, transverse components, $M_{x}, M_{y}$ of magnetization, in addition to a longitudinal component, $M_{z}$. According to the static

B2.1.4 
field solution of the Bloch equations, the magnitude of the transverse magnetization is $e^{-t / T_{2}(\vec{r})} M_{\perp}(\vec{r}, 0)$, which relates the $x$ and $y$ components through:

$$
\begin{aligned}
& M_{x}(\vec{r}, t)=e^{-t / T_{2}(\vec{r})} M_{\perp}(\vec{r}, 0) \cos \left(-\omega_{0} t+i \phi_{0}(\vec{r})\right) \\
& M_{y}(\vec{r}, t)=e^{-t / T_{2}(\vec{r})} M_{\perp}(\vec{r}, 0) \sin \left(-\omega_{0} t+i \phi_{0}(\vec{r})\right)
\end{aligned}
$$

where $-\omega_{0} t$ describes the phase generated from the rotation of the RF field and $T_{2}(\vec{r})$ is the usual $T_{2}$ decay. The phase $\phi_{0}(\vec{r})$ and magnitude $M_{\perp}(\vec{r}, 0)$ are determined by the initial RF pulse conditions.

The receive field laboratory components may be written quite generally in terms of the magnitude $\mathcal{B}_{\perp}$ and angle $\theta_{\mathcal{B}}(\vec{r})$ in the parametrization:

$$
\mathcal{B}_{x}^{\text {receive }} \equiv \mathcal{B}_{\perp} \cos \theta_{\mathcal{B}}(\vec{r}), \quad \mathcal{B}_{y}^{\text {receive }} \equiv \mathcal{B}_{\perp} \sin \theta_{\mathcal{B}}(\vec{r})
$$

Although one still needs the $z$ component of the magnetization along with the above Equation B2.1.7, the $z$ component does not effectively contribute to the emf. The key is that the longitudinal component does not rotate at the Larmor frequency but changes at a very slow rate as determined by $1 / T_{1}$. For static fields at the Tesla level, the Larmor frequency $\omega_{0}$ is at least four orders of magnitude larger than typical values of $1 / T_{1}$ and $1 / T_{2}$. Hence, the derivative of the $e^{-t / T_{1}}$ and $e^{-t / T_{2}}$ factors most certainly can be neglected, compared with the derivative of the $e^{-i \omega_{0} t}$ factor. (There are certain cases, such as in solids, where $T_{2}$ is on the order of a microsecond or less, and such approximations may no longer be valid.) With that approximation understood, the signal can be readily shown to behave according to:

$$
\text { signal } \propto \omega_{0} \int d^{3} r e^{-t / T_{2}(\vec{r})} M_{\perp}(\vec{r}, 0) \mathcal{B}_{\perp}(\vec{r}) \sin \left(\omega_{0} t+\theta_{\mathcal{B}}(\vec{r})-\phi_{0}(\vec{r})\right)
$$

The proportionality factor depends on amplifier gain and other factors, as determined by the detection scheme.

Equation B2.1.10 for the signal is easily modified for the more general situation. The replacement $T_{2} \rightarrow T_{2}{ }^{*}$ must be made in the presence of external field inhomogeneities, although this distinction is ignored in the current unit. A time-independent (or time-averaged) variation in the $z$ component of the local magnetic field, other than those already taken into account through $T_{2}^{\prime}$, may arise, for example, from the gradient fields used in imaging. These field variations change the precession frequency according to:

$$
\omega(\vec{r})=\omega_{0}+\Delta \omega(\vec{r})
$$

The correction $\Delta \omega(\vec{r})$ has been ignored in the outside factor $\omega_{0}$ in Equation B2.1.10, but if it cannot be omitted in the phase of the sinusoid. A simple, but relevant example is $\cos \left(10^{6}+\theta\right)$. Variations of $\theta$ in the interval $(0,2 \pi)$, though minuscule with respect to one million, can change the cosine value over the full range $(-1,1)$. Finally, in the case where additional fields have time dependence (e.g., when they are turned on and off), the phase $-i \omega(\vec{r}) t$ is replaced by $-i \int_{0}^{t} d t^{\prime} \omega\left(\vec{r}, t^{\prime}\right)$.

Radiofrequency Excitation and Reception

B2.1.5 
The limit where all spatial dependence can be neglected applies, for example, to studies of small homogeneous samples. Consider all quantities inside the integral in Equation B2.1.10 to be independent of $\vec{r}$. If the sample volume is $V_{s}$, then

$$
\text { signal } \propto \omega_{0} V_{s} e^{-t / T_{2}} M_{\perp} \mathcal{B}_{\perp} \sin \left(\omega_{0} t+\theta_{\mathcal{B}}-\phi_{0}\right) \quad \text { (space-independent limit) }
$$

For the precession frequency to be constant everywhere, the static field $B_{0}$ must also be uniform throughout space.

\section{Independent coils}

Whether $\overrightarrow{\mathcal{B}}$ receive pointed in the laboratory $x$-direction $\left(\theta_{\mathcal{B}}=0\right)$ or along the $y$-direction $\left(\theta_{\mathcal{B}}\right.$ $=\pi / 2$ ), or any other (spatially independent) direction in the $x-y$ plane, the received signal magnitude remains unchanged. Only the angle $\theta_{\mathcal{B}}$ changes in Equation B2.1.9. In this sense, one coil is as good as another, and two (or more) coils do not yield any more information. The signal from two uncoupled coils (coils that have small flux "linkage") can be used, however, to obtain an improvement in the ratio of signal to noise. The noise in one of these uncoupled coils is independent of the noise in the other whereas, for a single coil, assuming that more than one channel (the real or imaginary parts) of the single coil has been used to detect signal, the noise in one channel is the same as that in the other (i.e., the noise is correlated between the channels). For example, a circularly polarized (CP) receive coil satisfies the condition of having two independent channels. To the extent that the "cross-channel" noise is uncorrelated, the CP coil will increase the signal-to-noise ratio.

\section{Signal demodulation}

The rapid oscillations at the frequency $\omega_{0}$ in the above signal expressions are removed, in practice, by an electronic step of "demodulation," which is tantamount to viewing the signal from a rotating reference frame. Demodulation corresponds to the multiplication of the signal by a sinusoid or cosinusoid with a frequency at or near $\omega_{0}$. Strictly speaking, the transmit or "irradiation" RF frequency $\omega_{\mathrm{RF}}$ is this frequency, but in most experiments $\omega_{\mathrm{RF}}$ is chosen equal to $\omega_{0}$.

The frequencies of the original and demodulated signals are compared in Figure B2.1.2. In this figure, the original signal oscillates at Larmor frequency $\omega_{0}$ and a demodulation signal is applied at the frequency $\omega_{0}+\delta \omega$. After the demodulation, two frequencies appear, one at $\delta \omega$ and the other one at $2 \omega_{0}+\delta \omega$. Then a low-pass filter is applied to remove the signal at the higher frequency. In MR measurements, signals are demodulated and filtered before any image reconstruction is performed. (Usually, when people refer to the demodulated signal, they mean the demodulated and filtered signal.) After Fourier transforming the signal, an MR image is created.

\section{Free Induction Decay Signal}

The simplest MRI experiment involves detecting a global signal from a sample. Consider a $\pi / 2$-pulse applied uniformly in space to proton spins in a static magnetic field associated, say, with any hydrogen atoms present throughout the body (a macroscopic set). The pulse rotates the longitudinal magnetization (the excess spins) into the transverse plane after which the tipped spins freely and collectively precess. As discussed above, the total time-varying coherent magnetic field derived from the sum over all precessing proton spin fields would induce a small emf in any RF coil properly oriented to detect the corresponding flux changes. This experiment is called a free induction decay. The signal 


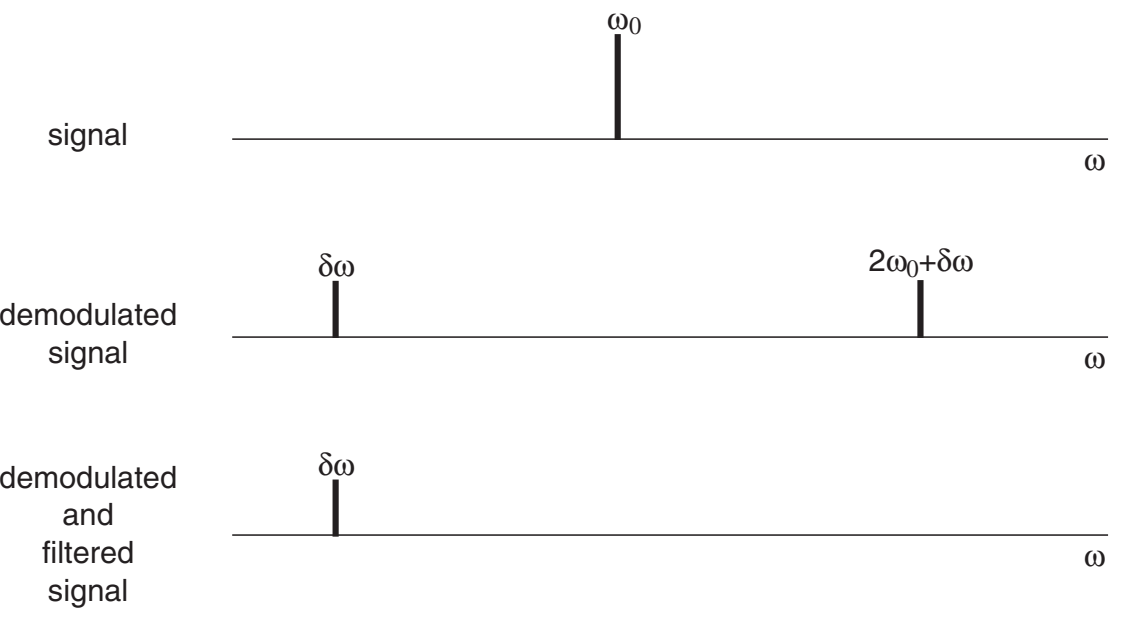

Figure B2.1.2 The shifts in frequency for signals from a uniform sample due to demodulation and filtering, with small offset $\delta \omega$. The thick bars represent the signal at the indicated frequencies.

An FID is performed routinely on MRI machines to tune RF coils and optimize system response. It can be used to locate the resonance peak for water and determine the RF amplitude and duration necessary to produce a maximum signal.

Consider the following simple FID experiment. Assume that the precession frequency is constant $\left(\omega_{0}\right)$ over all space and that $T_{2}$ is space-independent. The initial magnetization phase and $\theta_{\mathcal{B}}$ are assumed to be zero over the whole sample. After demodulation at $\omega_{0}$, Equation B2.1.10 becomes

$$
s(t) \propto \omega_{0} e^{-t / T_{2}} \int d^{3} r \mathcal{B}_{\perp}(\vec{r}) M_{\perp}(\vec{r}, 0)
$$

Equation B2.1.13 can be used to understand several examples of signal time dependence. In the laboratory frame, where no demodulation is applied, rapid oscillations at the frequency $\omega_{0}$ are damped by the relaxation factor, as shown in Figure B2.1.3A. Demodulation on-resonance removes the oscillatory behavior, leaving only the $T_{2}$ envelope (Fig. B2.1.3B). A demodulation slightly off-resonance leaves a low-frequency component, as shown in Figure B2.1.3C. Lastly, for a number of spins whose frequencies are slightly different from Larmor frequency, the total signal from those spins after demodulation yields a faster decay than caused by $T_{2}$ alone (Fig. B2.1.3D). The dephasing among the spin populations causes the reduced signal, resembling a $T_{2}^{\prime}$ effect and the overall signal shows a $T_{2}{ }^{*}$ decay-i.e., signal decays according to $e^{-t / T_{2}{ }^{*}}$ where $1 / T_{2}=1 / T_{2}+1 / T_{2}{ }^{\prime}$.

\section{Dependence on System Parameters}

Let us investigate what Equation B2.1.12 tells us about the variables on which the signal depends. Despite its lack of spatial information, this simple approximation is quite useful in understanding various imaging issues.

\section{Homogeneous limit}

Suppose that the equilibrium magnetization $M_{0} \hat{z}$ is independent of position (i.e., the sample is homogeneous), and it is uniformly rotated (i.e., the RF field is homogeneous) into the transverse plane with an on-resonance $\pi / 2$-pulse. Further, suppose that relaxation

Radiofrequency Excitation and Reception

B2.1.7 
A

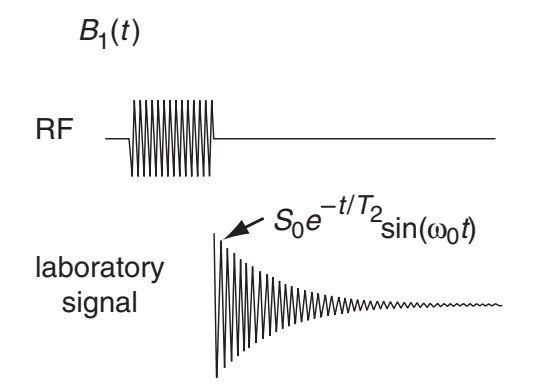

C

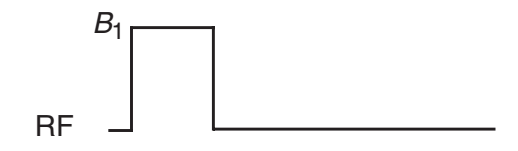

demodulated
signal

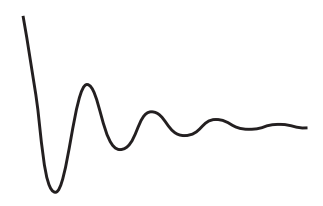

B
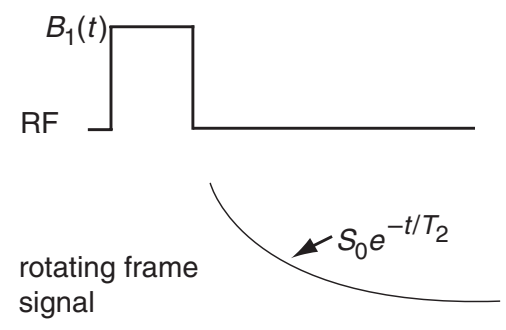

D

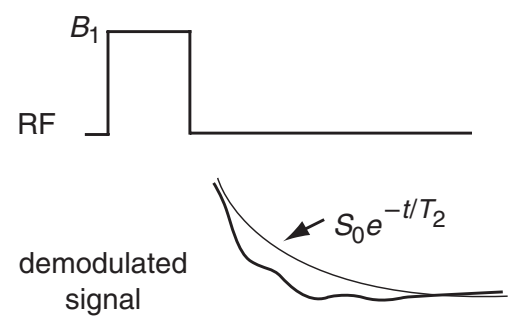

Figure B2.1.3 (A) The FID signal in the laboratory frame for all spins precessing at the same Larmor frequency. The laboratory RF transmit field oscillates at that frequency. (B) The same experiment but as measured in the Larmor rotating frame (i.e., demodulated). The rotating frame RF field is at "rest." (C) The demodulated FID signal when the demodulation is not exactly at the Larmor frequency. (D) The total demodulated FID signal from several isochromats, each with slightly different Larmor frequencies, exhibiting a decay, with slow oscillations, that is faster than $T_{2}$ decay alone, due to dephasing. The demodulation in panel $D$ is determined by the average (fast) frequency. Note that the laboratory signal in panel $\mathrm{A}$ is only suggestive since the oscillations seen in practice are too rapid to display. The $T_{2}{ }^{\prime}$ effects have not been included in any of the curves; note that the differences in the case shown in panel $\mathrm{D}$ could be considered, alternatively, to be due to field inhomogeneities.

effects may be neglected, and that the static field is also perfectly homogeneous. The proportionality in Equation B2.1.12 can be turned into an equation for the demodulated signal amplitude, if the electronic amplification factors are ignored:

$$
|s|=\omega_{0} M_{0} \mathcal{B}_{\perp} V_{s}
$$

Even in the presence of $T_{2}$ decay, and without demodulation, Equation B2.1.14 still represents the peak signal obtained at $t=0$ for a homogeneous sample. This signal from imaging human yields on the order of $1 \mathrm{mV}$.

Recall that $M_{0}$ is proportional to $B_{0}$ and $\omega_{0}=\gamma B_{0}$. Therefore, in the small sample limit, Equation B2.1.14 predicts a growth of $B_{0}^{2}$ in the signal as a function of field. A general analysis, including spatial dependence and differences in the sampled nuclei, is complicated, and electronic and sample noise must also be considered. For example, at high fields, noise also increases linearly with $B_{0}$ so that, by the above estimate, the more pertinent quantity, the signal-to-noise ratio, is only linearly proportional to $B_{0}$. It is the increase of signal-to-noise ratio with frequency that accounts for the interest in higher-

B2.1.8 
field imaging in MRI. Indeed, fields in excess of $10 \mathrm{~T}$ are already in use for NMR microscopy experiments and whole-body imaging systems are in operation up to $8 \mathrm{~T}$. Higher fields have additional attraction for spectroscopists, since the chemical shift dispersion increases linearly with field strength.

There are a number of technical difficulties associated with higher field imaging, however, and whether there is an optimal field strength for MRI remains to be seen. Optimal field strength is also likely to depend upon the application. The signal dependence on $V_{\mathrm{s}}$ is crucial to very high resolution imaging, since the signal in each voxel is limited by its volume and the available magnetization for the given field and temperature conditions. In general, as resolution is increased, the available signal decreases. This is easy to understand by considering the volume integral over the entire sample as a summation over smaller volumes (voxels, say) within the sample. The smaller the individual volume, the less signal it will produce. In order to obtain enough signal from the tiny volumes $(\approx 1000$ $\mu \mathrm{m}^{3}$ ) sought after by microscopists, fields of $\sim 7 \mathrm{~T}$ and higher are usually employed.

\section{Relative signal strength}

What we call the relative signal strength $\mathcal{R}$ of an MR experiment for a particular nuclear species can also be analyzed through Equation B2.1.14. (The more common definition for the sensitivity of an MR experiment is based on the signal-to-noise ratio of a given chemical species relative to a fixed standard species, usually ${ }^{1} \mathrm{H}$, which is normalized to have a sensitivity of unity.) The first step is to find the dependence of the equilibriummagnetization on the gyromagnetic ratio $\gamma_{i}$ and spin $s_{i}$ of a specific nucleus $i$ :

$$
M_{o_{i}} \propto a_{i} s_{i}\left(s_{i}+1\right) \gamma_{i}^{2}
$$

where $a_{i}$ is the natural abundance (the fractional occurrence of a given stable isotope relative to all stable isotopes). The relative signal strength $\mathcal{R}_{i}$ of a given nuclear species $i$ can be defined from the species-dependent factors in Equation B2.1.14 combined with Equation B2.1.15. Noting $\omega_{0} \propto \gamma$, we find:

$$
\mathcal{R}_{i} \equiv\left|\gamma_{i}\right|^{3} r_{i} a_{i} s_{i}\left(s_{i}+1\right)
$$

The weighting $r_{i}$ is the relative abundance in the human body of the given element referenced to some nucleus. In this text, signal strength is quoted relative to ${ }^{1} \mathrm{H}$ in gray matter.

Using $\gamma$ instead of $\omega_{0}$ means that only for the same static field can a comparison of two different elements be made through Equation B2.1.16. The parameters for several other elements relative to ${ }^{1} \mathrm{H}$ are presented in Table B2.1.1. Its sizable gyromagnetic ratio and large fractional presence explain why ${ }^{1} \mathrm{H}$ is the subject of choice for imaging of humans by nuclear magnetic resonance.

\section{Radiofrequency field effects}

Up to now, we have assumed that the transmit and receive RF coils produce uniform fields over the imaging volume. If this is not the case, the image intensity will vary as a function of position, even for a uniform sample. The image will appear to be brighter or darker in regions where either one or both of the RF fields, referred to by $B_{1}{ }^{\text {transmit }}$ (tips spins) or $\mathcal{B}^{\text {receive }}$ (measures signal), vary. The changes in image intensity as a function of field depend on flip angle and which field is being discussed. Assuming a uniform rotation by

Radiofrequency Excitation and Reception

B2.1.9 
the transmit coil, the image will be darker in regions where $\mathcal{B}^{\text {receive }}$ is smaller, and brighter where $\mathcal{B}^{\text {receive }}$ is larger.

The effect of the transmit field is more complex. Consider the case of a $\pi / 2$ flip angle. Any field which is greater or less than ideal (i.e., than that strength resulting in exactly a $90^{\circ}$ rotation), results in reduced transverse magnetization, and, therefore, a decrease in available signal. This statement is valid for a single pulse experiment only. For example, let us assume that two identical spins are excited by different transmit fields. The first spin experiences $B_{1}{ }^{\text {transmit }}$ (spin 1$)=C$, and receives a $\pi / 2$ flip. The second spin only experiences a field of $B_{1}^{\text {transmit }}(\operatorname{spin} 2)=C / 2$, and therefore is rotated by $45^{\circ}$. As a result, the signal (or the transverse magnetization) produced by the second spin is $1 / \sqrt{2}$ lower than the signal from the first spin.

These effects can lead to inhomogeneity in the image if the same coil is used for tipping the spins and detecting the signal, or if similar field profiles exist in $B_{1}^{\text {transmit }}$ and $\mathcal{B}^{\text {receive }}$. For example, if an RF field tips a spin by an angle $\theta$ from Equation B2.1.13, the signal is proportional to $\theta \sin \theta$. (The factor $\theta$ is from $\mathcal{B}$ receive and the factor $\sin \theta$ is from the projection of $M_{0}$ on the transverse plane.) Transmit and receive coils are carefully chosen in each imaging situation and designed to maximize image quality, but it is still necessary to be aware of coil effects when viewing an image.

\section{LITERATURE CITED}

Haacke, E.M., Brown, R.W., Thompson, M.R., and Venkatesan, R. 1999. Magnetic Resonance Imaging: Physical Principles and Sequence Design. John Wiley \& Sons, New York.

\section{KEY REFERENCES}

Beall, P.T., Amtey, S.R., and Kasturi, S.R. 1984. NMR Data Handbook for Biomedical Applications. Pergamon Press, New York.

This text has a more comprehensive list of active NMR elements (i.e., those with nonzero spin).

Haacke et al., 1999. See above.

This text covers the technical aspects presented here, but in more detail, and also discusses more advanced materials.

Hinshaw, W.S. and Lent, A.H. 1983. An introduction to NMR imaging: From the Bloch equation to the imaging equation. Proc. IEEE 71:338.

This article has a good introduction to basic NMR signal detection.

Hoult, D.I. and Richards, R.E. 1976. The signal-to-noise ratio of the nuclear magnetic resonance experiment. J. Magn. Reson. 24:71.

The basic interaction between the nuclear magnetic moment and the rf coil as well as the reciprocity concept are introduced in this widely quoted paper.

Jackson, J.D. 1975. Classical Electrodynamics. John Wiley \& Sons, New York.

This is an advance textbook of classical electromagnetism.

Contributed by Yu-Chung Norman Cheng and E. Mark Haacke

Case Western Reserve University

Cleveland, Ohio

and The MRI Institute for Biomedical Research

St. Louis, Missouri 